This is the peer reviewed version of the following article:

Vasquez-Sancho F., Abdollahi A., Damjanovic D., Catalan G.. Flexoelectricity in Bones. Advanced Materials, (2018). 30. 1705316: - . 10.1002/adma.201705316,

which has been published in final form at https://dx.doi.org/10.1002/adma.201705316. This article may be used for non-commercial purposes in accordance with Wiley Terms and Conditions for Use of Self-Archived Versions. 


\section{Flexoelectricity in bones}

Fabian Vasquez ${ }^{1,2}$, Amir Abdollahi ${ }^{3}$, Dragan Damjanovic ${ }^{4}$, Gustau Catalan ${ }^{1,5}$

${ }^{1}$ Institut Catala de Nanociencia i Nanotecnologia (ICN2), CSIC and the Barcelona Institute of Nanoscience and Nanotecnology (BIST). Bellaterra 08193, Barcelona, Catalonia.

${ }^{2}$ Centro de Investigación en Ciencia e Ingeniería de Materiales, Universidad de Costa Rica, San José 11501, Costa Rica.

${ }^{3}$ Laboratori de Calcul Numeric, Universitat Politecnica de Catalunya, Barcelona.

${ }^{4}$ Ecole Politechnique Federale de Lausanne (EPFL), Lausanne, Switzerland.

${ }^{5}$ Institut Catala de Recerca i Estudis Avançats (ICREA), Barcelona, Catalonia.

Remodeling of bones has been related to their electromechanical properties since Fukada and Yasuda's seminal measurement in 1957 of bone piezoelectricity ${ }^{1}$. It is believed that the piezoelectricity of collagen (the main structural protein of bones) is responsible for this effect ${ }^{2}$. However, since the discovery of flexoelectricity ${ }^{3,4}$, it has been known that strain gradients can also generate voltages in materials of any symmetry. Here we have measured the flexoelectricity of bone and bone mineral (hydroxyapatite), and determined that flexoelectricity accounts for most or all of the bending-induced polarization of bones. Knowing the flexoelectric coefficient of hydroxyapatite has also allowed us to calculate the stress-induced flexoelectric fields generated around cracks in bone mineral. The results indicate that crack-generated flexoelectricity is large enough to be able to induce osteocyte apoptosis and thus initiate the crack-healing process, pointing to a central role of flexoelectricity in bone damage repair and remodelling. 
All animals -including of course humans- require electricity to perform functions as basic as muscle contraction or nervous impulse sensing and transmission. In the case of vertebrates, electricity is also essential for bone regeneration ${ }^{5,6}$. One way to generate electricity is through piezoelectricity, which in bones can be provided by collagen ${ }^{2,7}$. In addition, ionic streaming potentials ${ }^{8}$ also contribute to the electromechanical properties of wet bones. Intriguingly, however, bone-repair functionality (osteoblast accumulation) has been observed near cracks at the surface of pure hydroxyapatite ceramics, where there is neither collagen nor streaming currents ${ }^{9}$. This result indicates that hydroxyapatite itself can also generate signals for the repairing cells. The nature and origin of such signals, however, is not known, and is one of the most intriguing and enduring problems in the field of osteogenesis $^{10-12}$.

One potential explanation is bone mineral piezoelectricity. Early studies suggested that hydroxyapatite is centrosymmetric and therefore not piezoelectric ${ }^{13}$, but more recent structural refinements ${ }^{14}$ suggest that it might be. However, functional measurements are ambiguous. Thin films yield substantial piezoelectric coefficients ${ }^{15}$, but thin films can easily become polarized by built-in fields, strain gradients, or defects ${ }^{16}$. Bulk ceramics, meanwhile, sometimes yield a small piezoelectricity ${ }^{17}$ and sometimes no piezoelectricity at all ${ }^{2}$. These variations probably reflect differences in sample composition or morphology, making it difficult to make definite statements about intrinsic properties. Our own hydroxyapatite ceramic and commercially acquired ceramics from Berkeley Advanced Biomaterials, Inc., were measured by a direct load method ${ }^{16}$, yielding piezoelectric coefficients smaller than of $0.001 \frac{\mathrm{pC}}{N}$. This is at least two orders of magnitude smaller than the piezoelectricity of bone ${ }^{7}$, and is comparable to the residual (defect-induced) piezoelectricity of $\mathrm{SrTiO}_{3}$, a reference non-piezoelectric material used for comparison (Supplementary materials Figure S 1). Our bone ceramics are therefore not significantly piezoelectric, a result consistent with the lack of piezoelectricity in de-collagenized bones $^{2}$. Macroscopic measurements of course do not rule out the existence of piezoelectricity on a microscopic level: piezoelectric grains with different orientation can in theory average out their aggregate contribution; however, piezo response force microscopy (Supplementary material Figure S 2 to Figure S 4) showed no phase contrast between grains. If we discard piezoelectricity, however, how does hydroxyapatite direct the activity of osteoblasts towards damaged regions ${ }^{9}$ ?

A plausible hypothesis is that bone mineral generates electromechanical signals due to flexoelectricity, which is a property of all dielectric (and even semiconductor ${ }^{18}$ ) materials whereby they polarize in response to an inhomogeneous deformation such as bending ${ }^{19}$. The combination of built-in structural flexibility and mechanical texture at the microscale -the scale in which cells operate and build- is inherent to biological tissues, and constitutes an optimal environment for flexoelectricity. 
For example, flexoelectricity has already been identified in stereocillia (inner ear micro-hairs), as an important ingredient of mammalian hearing ${ }^{20}$.The highly textured and inhomogeneous structure of bones, with radial porosity gradients and curved walls, also lends itself to flexoelectric phenomena. Already in 1975 Williams $^{21}$ claimed that some electromechanical properties of bones, could perhaps be explained by "gradient polarization" or inhomogeneous piezoelectricity. Around the same time, Lakes also performed a theoretical analysis of the potential role of gradients in bones which could not be substantiated due to lack of quantitative knowledge of their flexoelectric coefficients ${ }^{22}$. Later, Fu reported in a conference the existence of bending-induced polarization in bones ${ }^{23}$, wrongly attributing this flexoelectric-like response to collagen. Though these antecedents are few and scattered, together they provide tantalising evidence that there may be an important role for flexoelectricity in bones.

In this paper, we have quantified the flexoelectricity of hydroxyapatite and its participation in the electromechanical response of bones. The results indicate that most of the electromechanical response of a bone to bending comes from the flexoelectricity of bone mineral rather than from collagen. We have then used our measured flexoelectric coefficient of hydroxyapatite to calculate the flexoelectricity generated by cracks in bone mineral (see Figure 1). The calculated intensity exceeds $5 \frac{\mathrm{kV}}{\mathrm{m}}$ within a perimeter of $40 \mu \mathrm{m}$ around the crack tip, and it therefore can provide a powerful electrical signal from the centre of damage to stimulate bone repair.

Fresh bovine femurs were cut in beams oriented parallel to the bone axis and electroded for measuring flexoelectricity. The same femurs were also ground to powder, calcined and sintered into ceramic pellets (see Methods). We used a dynamic mechanical analyzer to deliver an oscillatory bending and a lock-in amplifier to detect the bending-induced polarization (see Methods). The bending-induced polarization of bone, natural hydroxyapatite, and commercially-acquired synthetic hydroxyapatite is shown in Figure 2. The effective flexoelectric coefficients $\mu_{e f f}$, are extracted from the slopes of the linear fits of the polarization as a function of bending (See Methods). 


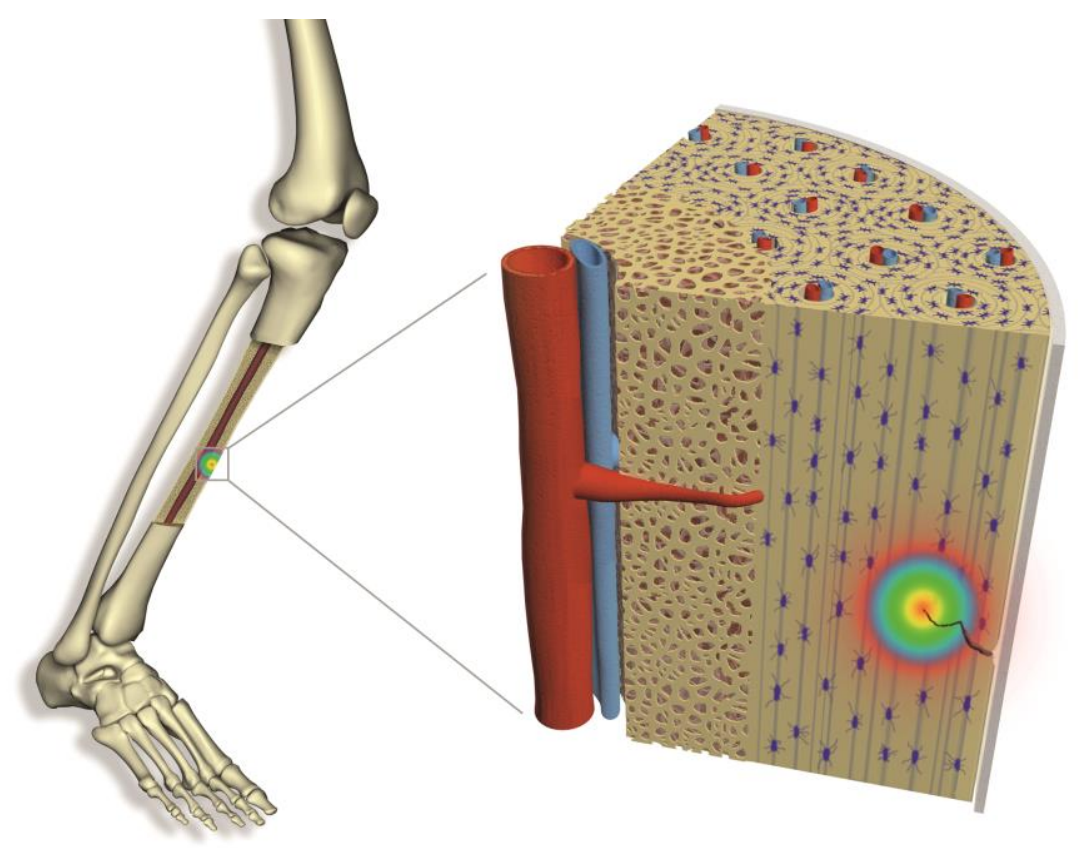

Figure 1: Strain gradients can be large around small defects such as micro-cracks in bone mineral, so gradientinduced electricity (flexoelectricity) is also expected to be large around such defects.

Bones and hydroxyapatite presented some variation from sample to sample. The dispersion of the flexoelectric coefficient for each material is presented as the shadowed area: red for hydroxyapatite and blue for bones. The effective flexoelectric coefficients are between $0.4-2.6 \frac{\mathrm{nC}}{\mathrm{m}}$ for bone, and between $0.7-1.6 \frac{\mathrm{nC}}{\mathrm{m}}$ for hydroxyapatite. Collagen increases the mechanical toughness of bones, allowing them to withstand bigger bending than brittle hydroxyapatite ceramics; but, for any given curvature, hydroxyapatite flexoelectricity is comparable to the flexoelectricity of bones. Hydroxyapatite flexoelectricity can by itself account for the bending-induced polarization of bones without needing to invoke collagen piezoelectricity. 


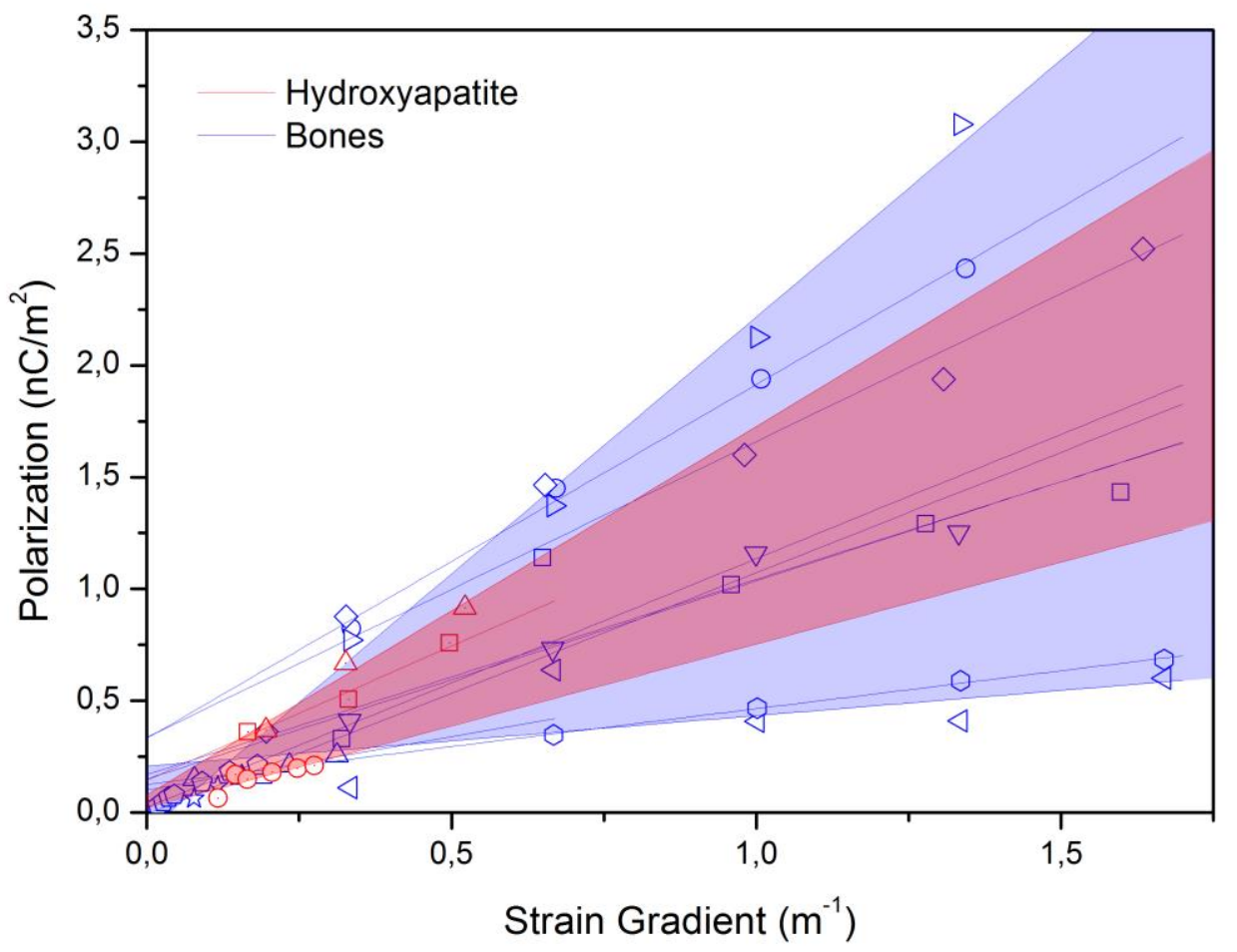

Figure 2. The flexoelectric coefficient is the constant of proportionality between a strain gradient (bending) and the bending-induced polarization. For greater accuracy, measurements were made for different applied forces (which induced different curvatures).The shadowed areas represent the dispersion of the data for bones (blue) and hydroxyapatite (red).

The next important question is: considering that bones already generate electromechanical voltages from streaming potentials and collagen piezoelectricity, what (if any) is the additional benefit of having a flexoelectric contribution from bone mineral? The answer appears to be related to the multiscale functional architecture of bones. Strain gradients grow in inverse proportion to feature $\operatorname{size}^{19,24}$. This means that although at macroscopic scales the average strain (and thus piezoelectricity) can dictate the global response, at small scales the strain gradient, and thus flexoelectricity, can be much larger and dominate the local electromechanical response ${ }^{23}$. A dramatic manifestation of this principle takes place at the apex of cracks, which concentrate in a very small volume (a crack junction is atomically sharp) the maximum stress that a material can withstand before rupture; according to theoretical calculations, the flexoelectric polarization near a crack apex can exceed the piezoelectric polarization for even the best piezoelectric materials ${ }^{25}$. In the context of bones, micro-cracks are common flaws formed due to cyclically applied stress, but they usually represent no risk for the integrity of the bone thanks to the process of remodelling ${ }^{11,26}$. As our calculations show, crackgenerated flexoelectricity is capable of triggering the process of damage repair and remodelling. 
The critical intensity factor $K_{C}$, which in bones is in the order of $3 \mathrm{MPa} \cdot \mathrm{m}^{1 / 227}$; this is the stress concentration at which cracks propagate through bone. Using our measured flexoelectric coefficients, we have calculated the flexoelectric field (Figure 3) around a micro-crack under critical load (see Methods). The flexoelectric field is biggest at the crack tip and decays progressively away, being bigger than $10^{3} \frac{\mathrm{V}}{\mathrm{m}}$ up to a distance $50 \mu \mathrm{m}$ around the crack apex. These numbers are significant because pulsed electric fields of $5 \frac{\mathrm{kV}}{\mathrm{m}}$ are known to induce apoptosis in bone cells ${ }^{28}$, osteocyte apoptosis being the first step of bone regeneration; when dead, osteocytes release chemical triggers that signal the osteoclasts to initiate the repair by cleaning the damaged region, followed by osteoblasts that segregate new bone mineral ${ }^{10,23}$. Electric fields also attract screening ions creating electrochemical gradients that assist osteogenesis ${ }^{29}$, thus further increasing the velocity of reparation of the damaged region ${ }^{30}$.

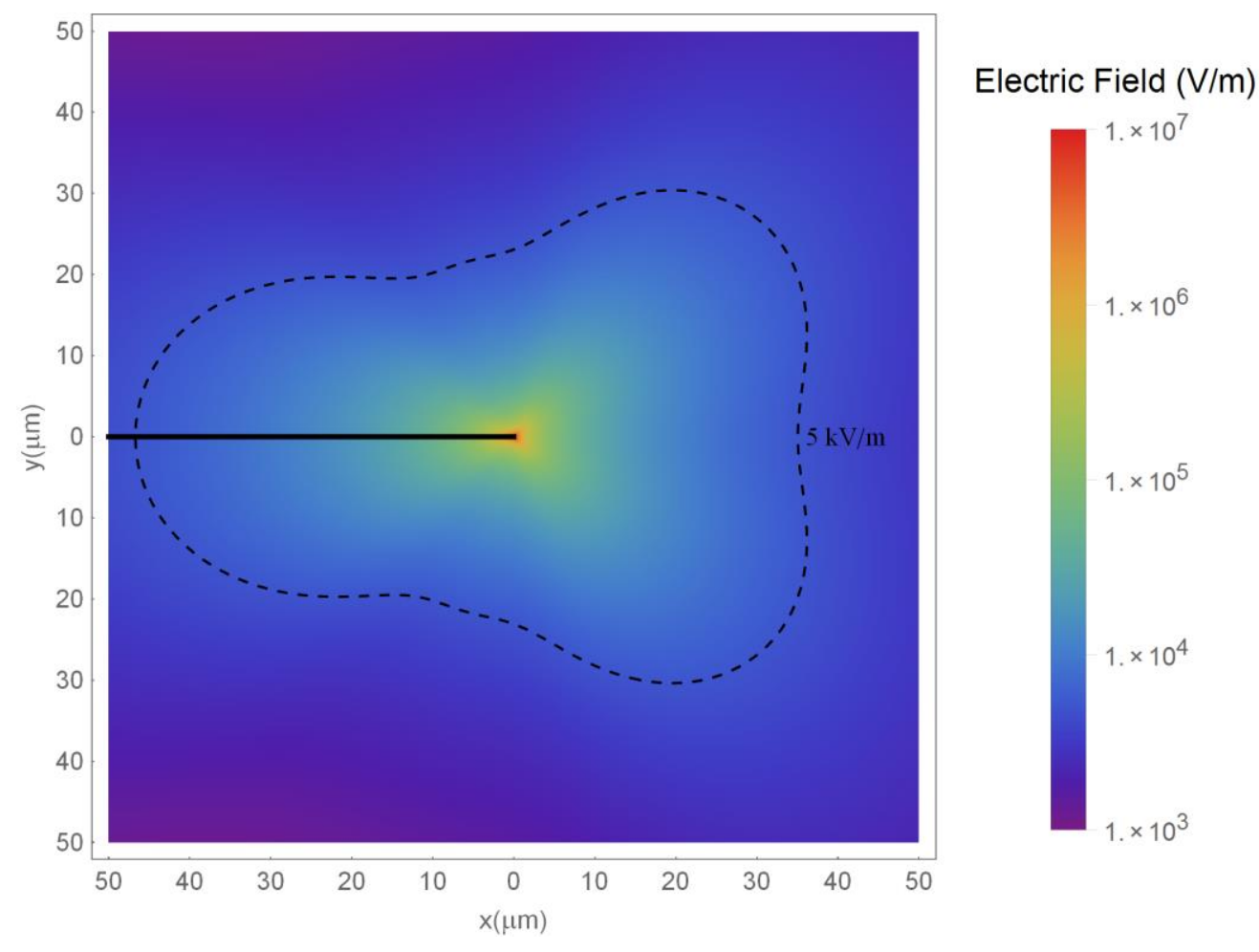

Figure 3. Calculated flexoelectric field distribution around a micro-crack in bone mineral. The dashed line marks the region where the field is strong enough to be able to induce osteocyte apoptosis.

Osteoblast tend to attach near by the tip of cracks in pure bone mineral ${ }^{9}$, suggesting that osteoblasts do indeed detect a crack tip as the centre of damage. Moreover, the apex is itself a movable entity: as the crack is healed, its apex will recede, continually pointing to the osteoclasts and osteoblasts the new position of the region to repair ${ }^{10}$. Flexoelectricity is strong enough to act as the beacon in this process, and this result suggests a new line of inquiry for tissue regeneration where 
gradient engineering could be used as an additional degree of freedom in bone-forming prosthetic designs.

\section{Methods}

Freshly cut (less than 48 hours from slaughter) bovine femurs were obtained from a butcher's shop and stored in a physiological solution. Pieces of cortical bone were then cut using a diamond wire at low speed in order to avoid damage to the tissue. The samples were cut in consideration of the orientation of the collagen inside the bone; in this case, all the samples were longitudinal to the long axis of the bone. The samples were polished up to $0.1 \mu \mathrm{m}$ grain size disc with an Allied precision polishing system at low velocity to minimize damage to the samples.

Hydroxyapatite compact discs were commercially obtained from Clarkson Chromatography Products, INC., with certified purity greater than $95 \%$. Also we produced our own hydroxyapatite from bovine bones following the procedure of Ooi,C. et al. ${ }^{31}$. In order to do the compact discs we milled the hydroxyapatite and sieved the powder to $125 \mu \mathrm{m}$ particle size. Then, the powder was uniaxially pressed into pellets of $22.5 \mathrm{~mm}$ of diameter with 25 metric tons. Finally the pellets were air sintered at $1360{ }^{\circ} \mathrm{C}$ during 4 hours. Samples were cut and polished using the same procedure as for the bones.

Polarization was induced by a DMA8000 dynamic mechanical analyser (DMA) of Perkin-Elmer and was measured using the method described by Zubko et al. ${ }^{32}$. The DMA was used to apply a periodic three-point bending stress at room temperature. This periodic signal was used as a reference for a lock-in amplifier, model 830 of Stanford Research Instruments, while the signal obtained from the electrodes fed the measurement channel of the lock-in amplifier, which recorded the bending-induced

displacement currents. The current was converted into polarization using $P=\frac{I}{2 \pi v A}$, where $v$ is the frequency of the bending force and $A$ is the area of the electrodes. The polarization measured by the lock-in is related to the effective flexoelectric coefficient $\mu_{e f f}$ by

$$
\overline{P_{3}}=\mu_{13}^{e f f} \overline{\frac{\partial \varepsilon_{11}}{\partial x_{3}}} \text { and } \overline{\frac{\partial \varepsilon_{11}}{\partial x_{3}}}=\frac{12 z_{0}}{L^{3}}(L-a)
$$

where $L$ is the separation between the standing points of the sample, $a$ is the half-length of the electrodes, and $z_{0}$ is the maximum vertical deflection in the middle of the sample. Typical values used in our measurements were $L=12 \mathrm{~mm}, a=2 \mathrm{~mm}$ and $z_{0}=2 \mu \mathrm{m}$. Measurements were taken after all samples had been dried in an oven at a temperature of $90{ }^{\circ} \mathrm{C}$ for 7 hours.

From equation (1), the effective flexoelectric coefficient is defined as the relation between the polarization and the stress gradient. For more accuracy, several strain gradients were applied to each 
sample and the flexoelectric coefficient was extracted from the slope of the plots of polarization as a function of strain gradient ${ }^{19}$, as can be seen in Figure 4.

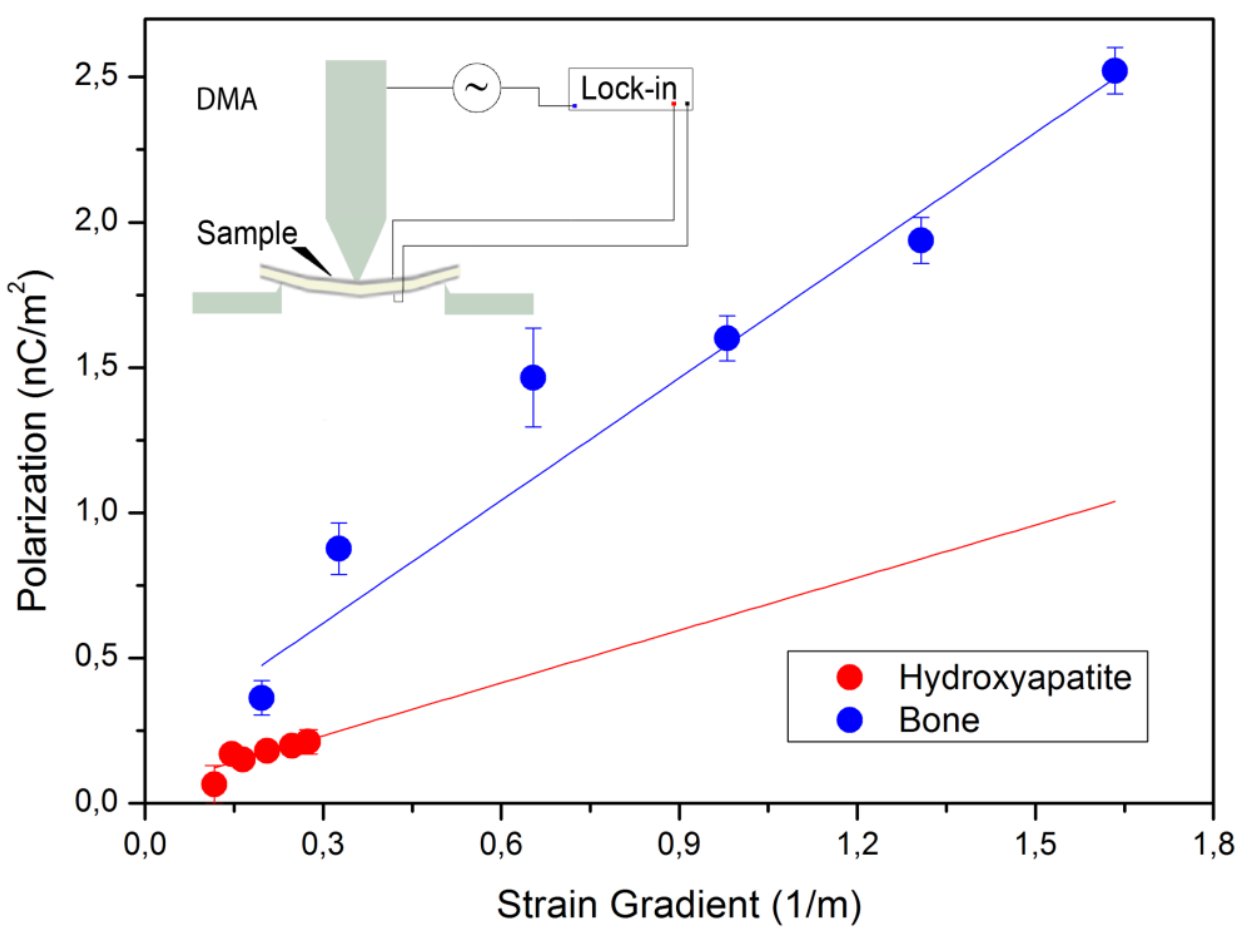

Figure 4: Flexoelectric coefficients were calculated as the slopes of the linear fits between the curvature and the bending-induced polarization. Because bones are more flexible than hydroxyapatite, it could withstand much larger curvatures, but the slope was still almost the same as for pure hydroxyapatite. Inset: sketch of the measurement apparatus.

Calculation of the flexoelectric field was made from the equations of strain around a crack mode $1^{33}$

$$
\varepsilon_{\mathrm{ij}}^{\mathrm{el}}=\frac{1+v}{\mathrm{E}} \sigma_{\mathrm{ij}}-3 \frac{\mathrm{v}}{\mathrm{E}} \sigma_{\mathrm{m}} \delta_{\mathrm{ij}}
$$

where $\sigma_{\mathrm{ij}}$ is the stress applied to the crack in each direction:

$$
\begin{gathered}
\sigma_{11}=\frac{\mathrm{K}_{\mathrm{I}}}{\mathrm{Y}_{11} \sqrt{2 \pi \mathrm{r}}} \cos \frac{\theta}{2}\left(1-\sin \frac{\theta}{2} \sin \frac{3 \theta}{2}\right) \\
\sigma_{22}=\frac{\mathrm{K}_{\mathrm{I}}}{\mathrm{Y}_{22} \sqrt{2 \pi \mathrm{r}}} \cos \frac{\theta}{2}\left(1+\sin \frac{\theta}{2} \sin \frac{3 \theta}{2}\right) \\
\tau_{12}=\frac{\mathrm{K}_{\mathrm{I}}}{\mathrm{Y}_{12} \sqrt{2 \pi \mathrm{r}}} \cos \frac{\theta}{2} \sin \frac{\theta}{2} \cos \frac{3 \theta}{2},
\end{gathered}
$$

where $\mathrm{K}_{\mathrm{I}}$ is the intensity factor taken as $3 \mathrm{MPam}^{-\frac{1}{2}}$, and $\mathrm{Y}_{\mathrm{ij}}$ is the Young's modulus in the different directions. For the calculations $\mathrm{Y}_{11}=6 \mathrm{GPa}$ and $\mathrm{Y}_{22}=20 \mathrm{GPa}$, both values obtained from our measurements. Equations were transformed to Cartesians coordinates in order to compute the flexoelectric field:

$$
\mathrm{E}_{1}=\mathrm{f}_{11} \frac{\partial \varepsilon_{11}}{\partial \mathrm{x}_{1}}+\mathrm{f}_{12} \frac{\partial \varepsilon_{22}}{\partial \mathrm{x}_{1}}
$$




$$
\begin{gathered}
E_{2}=f_{22} \frac{\partial \varepsilon_{22}}{\partial x_{2}}+f_{21} \frac{\partial \varepsilon_{11}}{\partial x_{2}} \\
E=\sqrt{E_{1}^{2}+E_{2}^{2}},
\end{gathered}
$$

and $f_{i j}$ is the flexocoupling tensor. The flexocoupling tensor was calculated with the effective flexoelectric coefficient $\mu_{\mathrm{eff}}$ and the dielectric constant of bone $\epsilon$

$$
\mu_{\text {eff }}=\mathrm{f}_{\text {eff }} \epsilon
$$

For this calculation, $\mathrm{f}_{11}=\mathrm{f}_{22}=\mathrm{f}_{12}=\mathrm{f}_{21}=\mathrm{f}_{\text {eff }}=10 \mathrm{~V}$ and the shear component was taken as null.

\section{References}

1. $\quad$ Fukada, E. \& Yasuda, I. On the Piezoelectric Effect of Bone. J. Phys. Soc. Japan 12, 1158 1162 (1957).

2. Marino, A. A., Becker, R. O. \& Sidney, S. C. Origin of piezoelectric effect in Bone. Calc. Tiss. Res. 8, 177-180 (1971).

3. Kogan, S. M. Piezoelectric effect during inhomogeneous deformation and acoustic scattering of carriers in crystals. Sov. Phys. Solid State 5, (1964).

4. Bursian, E. \& Zaikovskii, O. I. Changes in curvature of ferroelectric film due to polarization. Sov. Phys. Solid State 10, 1121 (1968).

5. Bassett, C. A. L. \& Becker, R. O. Generation of Electric Potentials by Bones in Response to Mechanical Stress. Science 137, 1063-1064 (1962).

6. Spadaro, J. A. Mechanical and Electrical Interactions in Bone Remodeling *. Bioelectromagnetics 18, 193-202 (1997).

7. Fukada, E. \& Yasuda, I. Piezoelectric Effects in Collagen. Jpn. J. Appl. Phys. 3, 502B-502B (1964).

8. Gross, D. \& Williamsi, W. S. Streaming Potential and the Electromechanical Response of Physiologically-Moist Bone. J. Biomech. 15, 277-295 (1982).

9. Shu, Y. et al. Surface microcracks signal osteoblasts to regulate alignment and bone formation. Mater. Sci. Eng. C. Mater. Biol. Appl. 44, 191-200 (2014).

10. Verborgt, O., Gibson, G. J. \& Schaffler, M. B. Loss of Osteocyte Integrity in Association with Microdamage and Bone Remodeling After Fatigue In Vivo *. J. Bone Miner. Res. 15, 60-67 (2000).

11. Taylor, D., Hazenberg, J. A. N. G. \& Lee, T. C. Living with cracks : Damage and repair in human bone. Nat. Mater. 6, 263-268 (2007).

12. Lanyon, L. E. Osteocytes, Strain Detection, Bone Modeling and Remodeling. Calcif. Tissue Int. 53, 102-107 (1993).

13. Kay, M. I. \& Young, R. A. Crystal Structure of Hydroxyapatite. Nature 204, 1050-1052 (1964).

14. Haverty, D., Tofail, S. a. M., Stanton, K. T. \& McMonagle, J. B. Structure and stability of hydroxyapatite: Density functional calculation and Rietveld analysis. Phys. Rev. B 71, 94103 (2005).

15. Lang, S. B. et al. Ferroelectric polarization in nanocrystalline hydroxyapatite thin films on silicon. Sci. Rep. 3, 2215 (2013). 
16. Biancoli, A., Fancher, C. M., Jones, J. L. \& Damjanovic, D. Breaking of macroscopic centric symmetry in paraelectric phases of ferroelectric materials and implications for flexoelectricity. Nat. Mater. 14, 224-229 (2015).

17. Tofail, S. a M. et al. Direct and ultrasonic measurements of macroscopic piezoelectricity in sintered hydroxyapatite. J. Appl. Phys. 105, (2009).

18. Narvaez, J., Vasquez-Sancho, F. \& Catalan, G. Enhanced flexoelectric-like response in oxide semiconductors. Nature 538, 219-221 (2016).

19. Zubko, P., Catalan, G. \& Tagantsev, A. K. Flexoelectric Effect in Solids. Annu. Rev. Mater. Res. 43, 387-421 (2013).

20. Breneman, K. D., Brownell, W. E. \& Rabbitt, R. D. Hair cell bundles: flexoelectric motors of the inner ear. PLoS One 4, e5201 (2009).

21. Williams, W. S. \& Breger, L. Piezoelectricity in tendon and bone. Biomechanics 8, 407-413 (1975).

22. Lakes, R. The Role of Gradients Effects in the Piezoelectricity of Bone. IEEE Trans. Biomed. Eng. BME-27, 282-283 (1980).

23. Fu \& John. Experimental studies of the direct flexoelectric effect in bone materials. Bull. Am. Phys. Soc. (2010).

24. Maranganti, R., Sharma, N. D. \& Sharma, P. Electromechanical coupling in nonpiezoelectric materials due to nanoscale nonlocal size effects: Green's function solutions and embedded inclusions. Phys. Rev. B 74, 14110 (2006).

25. Abdollahi, A. et al. Fracture toughening and toughness asymmetry induced by flexoelectricity. Phys. Rev. B 92, 94101 (2015).

26. Lee, T. C., Staines, A. \& Taylor, D. Bone adaptation to load: microdamage as a stimulus for bone remodelling. J. Anat. 201, 437-46 (2002).

27. Norman, T., Vashishtht, D. \& Burr, D. Fracture Toughness of human bone under tension. $J$. Biomech. 28, (1994).

28. S. D. McCullen, J. P. McQuilling, R. M. Grossfeld, J. L. Lubischer, L. I. Clarke, E. G. Loboa, Application of low-frequency alternating current electric fields via interdigitated electrodes: effects on cellular viability, cytoplasmic calcium, and osteogenic differentiation of human adipose-derived stem cells. Tissue Eng., Part C 16, 1377 (2010).

29. Hammerick, K. E., James, A. W., Huang, Z., Prinz, F. B. \& Longaker, M. T. Pulsed direct current electric fields enhance osteogenesis in adipose-derived stromal cells. Tissue Eng. Part A 16, 917-31 (2010).

30. Rajabi, A. H., Jaffe, M. \& Arinzeh, T. L. Piezoelectric materials for tissue regeneration: A review. Acta Biomater. 24, 12-23 (2015).

31. Ooi, C. Y., Hamdi, M. \& Ramesh, S. Properties of hydroxyapatite produced by annealing of bovine bone. Ceram. Int. 33, 1171-1177 (2007).

32. Zubko, P., Catalan, G., Buckley, A., Welche, P. \& Scott, J. Strain-Gradient-Induced Polarization in SrTiO3 Single Crystals. Phys. Rev. Lett. 99, 167601 (2007).

33. Lawn, B. Fracture of brittle solids. (Cambridge University Press, 1993). 


\section{Supplementary materials}

1) Direct piezoelectric measurements of hydroxyapatite

Piezoelectric response was measured for commercially acquire hydroxyapatite used in the paper measurements in order to discard the possibility of a considerable piezoelectric contribution of hydroxyapatite in the electromechanical signal of bones.

Samples were subjected to cyclic loads of $30 \mathrm{~N}$ peak to peak, while the charge was measured with an oscilloscope. As a reference, Ba-doped $\mathrm{SrTiO}_{3}$, a centrosymmetric material with residual piezoelectric coefficient is presented. Both materials have a piezoelectric coefficient smaller than $0.001 \frac{p C}{N}$. Previous studies on piezoelectricity of human bones showed piezoelectric coefficients of $0.067 \frac{\mathrm{pC}}{\mathrm{N}}$. The piezoelectric coefficient of the hydroxyapatite used is, at least, one order of magnitude smaller than the piezoelectric coefficient of bones.
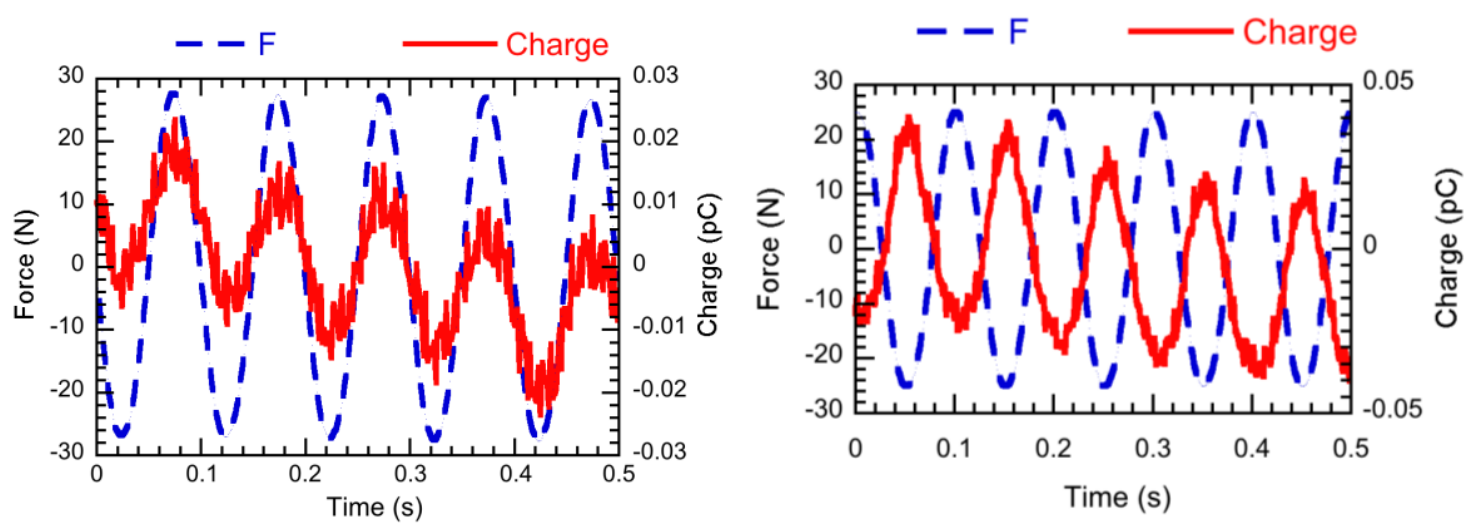

Figure S 1: Piezoelectric measurements of hydroxyapatite (left) and Ba-doped $\mathrm{SrTiO}_{3}$ (right). The piezoelectric coefficient is obtained by dividing the peak charge by the peak force applied.

2) Piezoresponse force microscopy measurements of hydroxyapatite

Single frequency Piezo response force microscopy was done to the samples measured with the DMA in order to find if there were differences in the piezo response of each grain of the sample. We used an EMF tip with a spring constant of $1.43 \frac{\mathrm{nN}}{\mathrm{mm}}$ and drive amplitude of $3 \mathrm{~V}$. 


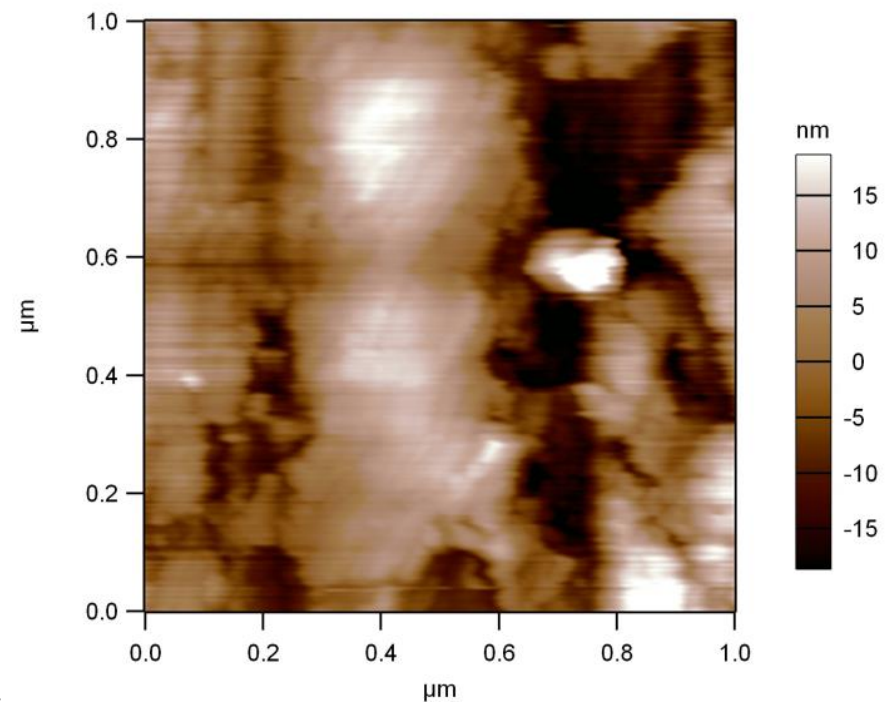

Figure S 2: Topography image of a surface of hydroxyapatite, showing grains and pores as expected from a polycrystalline ceramic.

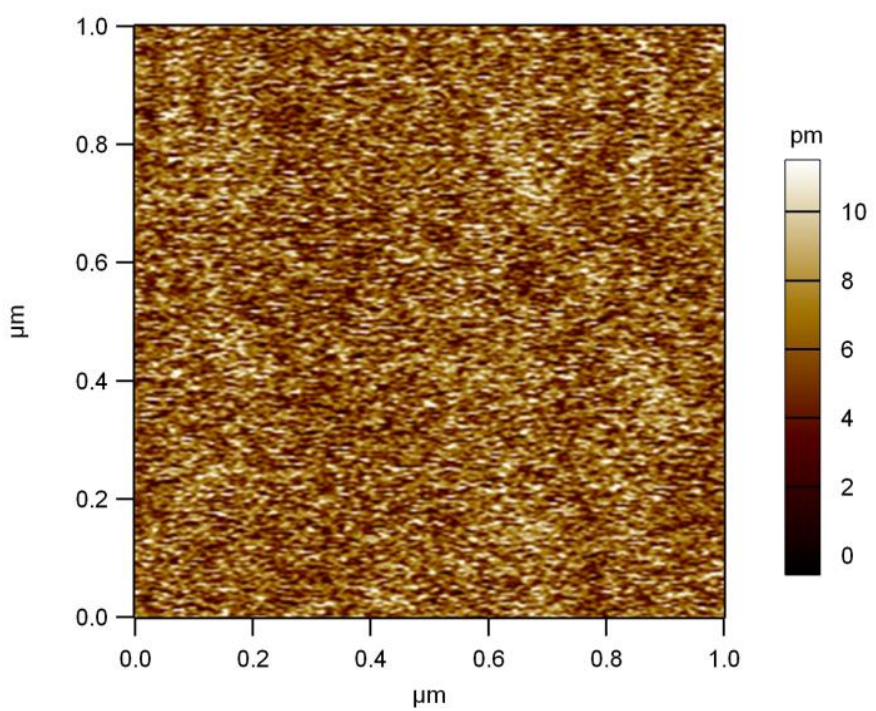

Figure S 3: Amplitude of the piezoelectric response of the surface of hydroxyapatite, showing no contrast between grains. 


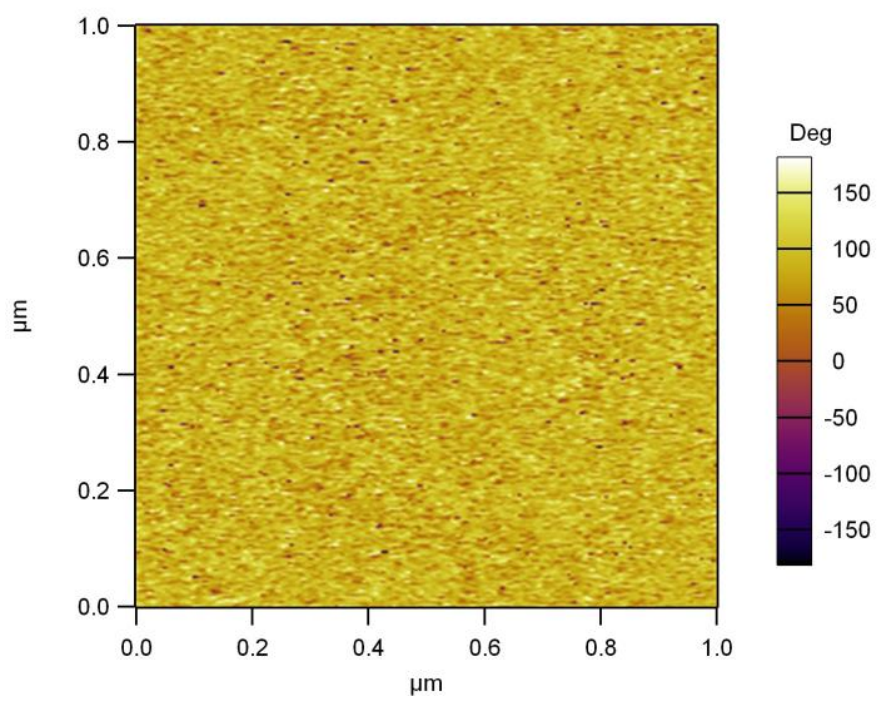

Figure S 4: Phase of the piezoelectric response of the surface of hydroxyapatite, showing no contrast between grains.

Figure S2, shows the topography of the surface of the sample where a big grain can be observed, next to two more grains. The amplitude and phase of the piezoresponse signal did not show contrast between the grains, meaning that, at the microscopic level, grains do not present piezoelectricity that could average out the macroscopic signal. The other possible scenario is that polarization of individual grains sums up producing a net, macroscopic polarization; this is consistent with the macroscopic piezoelectric measurements which presented a negligible signal. 